\title{
Estado del Arte sobre Métodos para Modelar la Arquitectura de Datos de un Sistema de Información
}

\section{State of the Art of the Methods for Modelling Data: Architecture of an Information System}

\author{
Tino Eduardo Reyna Monteverde ${ }^{1 *}$ \\ ${ }^{1}$ Facultad de Ingeniería Industrial y de Sistemas de la Universidad Nacional de Ingeniería, Lima, Perú
}

\begin{abstract}
RESUMEN
En este artículo se detallan las investigaciones más recientes respecto al tema del presente trabajo de investigación, "Método de Modelado de Arquitectura de Datos que asegure Robustez y Estabilidad". La estructura del artículo se ha organizado en dos bloques principales:

A. Modelado de Datos

B. Métodos de Modelado de Datos

Estos temas constituyen las líneas maestras de la presente investigación. Determinar con claridad el concepto y actividad de modelado es fundamental para soportar todo el desarrollo del método. El análisis de datos es otro de los temas críticos, por la misma naturaleza de esta etapa que no es operativa sino reflexiva. Muchas veces el personal técnico la evita por ser tediosa de allí la importancia de hallar alguna técnica rigurosa y sobre práctica, pero que posea sentido para el área de negocios. Finalmente como todo sistema de información comercial va dirigido hacia la empresa, se debe tomar en cuenta los patrones empresariales para fusionarlo con el modelo tecnológico.
\end{abstract}

Palabras clave.- Métodos, Modelando, Arquitectura, Datos, Sistemas de información de gestión.

\section{ABSTRACT}

This paper details the latest research on the subject of this research, "Method of Data Architecture Modelling to ensure robustness and stability". The structure of the paper is organized in two main blocks:
A. Data Modelling
B. Methods of Data Modelling

These issues are the main elements of the present investigation. Clearly identify the concept and modelling activity is critical for support the whole development of the method. Data analysis is one of the critical issues, by the very nature of this stage is not operational but thoughtful. Many times, technical staff often avoids it because is tedious, hence the importance of finding a rigorous and practical technique, which possessing sense for the business area. Finally, as all trade information system is directed towards the company, then you should take into account the business patterns to merge with the technological model.

Key words.- Methods, Modeling, Architecture, Data, Information systems management.

\section{INTRODUCCIÓN}

En esta investigación se detallan los artículos ordenados por afinidad temática, respecto al cuerpo de conocimiento del presente trabajo de investigación "Método de Modelado de Arquitectura de Datos que asegure Robustez y Estabilidad". La estructura del artículo se ha organizado en los bloques siguientes:
A.-
Modelado de Datos
B.-
Métodos de Modelado de Datos

En primer lugar determinar con claridad el concepto y actividad de Modelado, es fundamental porque es lo que soportará todo el desarrollo del método. Dado que la robustez se basa en la existencia de los atributos requeridos por el negocio, es fundamental el tema de los Requerimientos funcionales. Se debe tratar el tema crítico del tratamiento analítico de Datos, de manera que se encuentre alguna técnica rigurosa y sobre todo posea sentido respecto al área de negocios.

Finalmente como todo sistema de información comercial va dirigido hacia la empresa, luego se debe tomar en cuenta los patrones empresariales (Modelado conceptual de la empresa) para fusionarlo

La Revista Científica TECNIA protege los derechos de autor bajo la Licencia 4.0 de Creative Commons: Attribution 4.0 International (CC BY 4.0).

* Corresponding:

E-mail: treyna@uni.edu.pe 

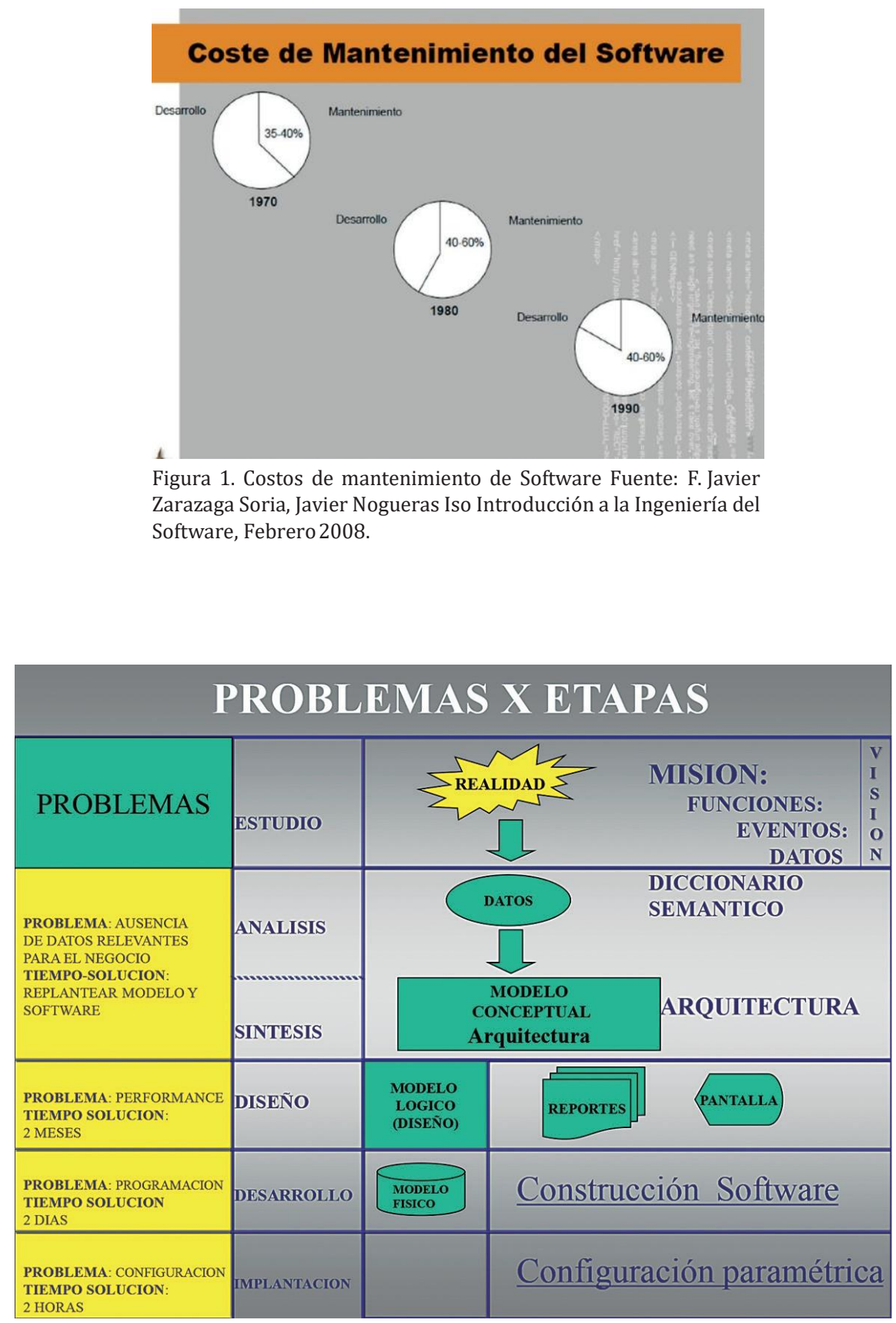

Figura 2. Problemas en cada etapa de proyecto (fuente propia). Problemas de análisis causan los mayores costos.

con el modelo tecnológico.

\section{¿Por qué buscar mejores métodos?}

La respuesta cae de madura, sobre todo para aquellos profesionales que conviven con los problemas graves de mantenimiento de sistemas, donde se tratan de sistemas que poseen grandes volúmenes de datos.
De acuerdo con Boehm [1] respecto a la eliminación de errores encontrado durante la etapa de requerimientos sería 3.5 veces si se eliminan durante la etapa de diseño o 50 veces durante la implementación o 170 veces durante la etapa de entrega (en producción).

Estudios empíricos muestran que si se realizan esfuerzos para asegurar calidad durante etapas previas a la implementación el costo efectivo podrá 
ser hasta 33 veces más si realiza en la implantación. Ver Figura 1 y 2

\section{ANTECEDENTES}

Cuando se utiliza al término Modelo de Datos es inevitable hacer referencia al Modelo ER (1973) que ha sido la base para diversas metodologías sobre análisis y diseño de sistemas, herramientas de ingeniería de software asistida por computador y repositorios de sistemas. En este sentido los términos "Modelo de Entidad-Relación (Modelo ER)", "Diagrama de Entidad-Relación (Diagrama ER)” y "Peter Chen" son utilizados habitualmente en textos de aprendizaje y en especificaciones de productos comerciales.

En 1982 McCarthy publicó en base al modelo ER el enfoque REA creado para el dominio de los sistemas contables, una propuesta que fue bien acogida y, ha sido fundamento para siguientes investigaciones.

En 1989 James Martin considerado uno de los gurús de la base de datos, publicó su obra "Sistemas de Información" donde expuso su nuevo enfoque de la Ingeniería de la Información diametralmente opuesto a la Ingeniería de Software. Sostiene que el núcleo de todo sistema de información es su base de datos la denomina "Piedra Fundamental Estable" para denotar el carácter Sólido y fundamental que juega el Modelo de datos en un sistema de Información.

Basado en estos antecedentes se expondrá la literatura afín al tema de investigación con el objetivo de conseguir Robustez y Estabilidad del modelo.

\section{REVISIÓN DE LITERATURA}

En esta sección se presenta los artículos ligados a los temas centrales de la presente investigación en orden temático para determinar la secuencia y tendencia proyectada.

\section{MODELADO DE DATOS}

[2] Data modeling: Description or design?. Graeme Simsion, Simon K. Milton, Graeme Shanks. Information \& Management, In Press, Corrected Proof, Febrero 2012.

Un Modelo es el resultado de la actividad de modelar, la misma que consiste en determinar los elementos básicos de un sistema y las relaciones entre ellos. Tanto los elementos como sus relaciones son descubiertos por el analista, de manera objetiva, es decir lo que él descubra lo debería hacer cualquier otro analista que aplique correctamente el análisis. Es explicitar en un documento lo que no se percibe directamente por nuestros sentidos, sino apelando a la capacidad de abstracción.

Por otro lado la actividad de diseño es muy diferente, al plantear soluciones totalmente subjetivas del sistema.

El sistema debe ser diseñado antes de ser construido, durante el diseño se determinan todas las características que va a poseer el sistema. El autor considera que en el proceso del modelado también se realiza diseño, dado que toda persona tiene su particular punto de vista, de alguna manera indirecta se evidencia en el resultado final.

En la exacta aplicación del concepto de modelar, que es descubrir la estructura oculta y subyacente, luego no es posible adicionar perspectiva personal porque se trata de un enfoque analítico. Toda esta confusión tiene su origen entre lo que se considera Ingeniería y Arquitectura. La Ingeniería tiene por misión la optimización del sistema sobre las limitaciones dadas por el modelo funcional del área de negocios. Mientras que la Arquitectura tiene libertad de proponer esquemas nuevos o modificaciones al sistema (resultado del descubrimiento), es decir tiene un enfoque de gestor o iniciador de una nueva realidad. Se entiende que la libertad es relativa no es absoluta, es decir es libre dentro de un rango finito determinado por parámetros bien definidos y precisos.

Por ejemplo los investigadores de la rama biológica llegaron a descubrir el modelo de la molécula del ADN. Este modelo presenta los elementos que lo constituyen y el tipo de relación que los une. Se ha descubierto la Arquitectura de la molécula del ADN.

\section{COMENTARIO}

La actividad de modelado conceptual es encontrar la Arquitectura al descubrir la esencia del modelo extraído de una realidad. El Arquitecto traduce lo que la realidad transmite a través de mensajes implícitos que deben ser explicitados con exactitud por parte del analista, lo cual asegura plantear requerimientos no definidos por el usuario, que subyacen ocultos. En este punto cabe una reflexión respecto al paradigma del Arquitecto y del Ingeniero. Típicamente la formación del profesional en ingeniería es buscar la solución óptima al escenario entregado, vale decir no debe buscar lo mejor sino lo óptimo (lo mejor con lo 
que cuenta en el momento). Por el lado del Arquitecto tiene la libertad de hacer propuestas bajo ciertos parámetros indicados por él Sistema y el entorno de la misma.

\section{Modelar es descubrir (su naturaleza) no diseñar.}

\section{Características esenciales del Modelo Conceptual [3]}

Conceptual Data Modelling: An Empirical Study of Expert and Novice Data Modellers Graeme Shanks Department of Information Systems, Monash University Melbourne, Australia, May 1997.

Las características deseables de una arquitectura de un modelo de datos son las siguientes:

a. Sintaxis correcta.- Se debe respetar toda la nomenclatura

b. Semánticamente completo.- Se debe definir todos los atributos que el negocio necesite como información requerida.

La métrica para esta característica se da en función de dos variables.

E1 $=$ Errores mayores

$\mathrm{E} 2=$ Errores menores

$\mathrm{N}$ = Número de componentes del modelo.

Para aplicar esta métrica se requiere comparar el modelo correcto contra el modelo a validar. Un error mayor se considera cuando está ausente una entidad o una relación. Mientras que un error menor se considera cuando se ha caído en redundancia o errores de cardinalidad. Por cada error mayor se penaliza con 1 y por un error menor con 0.3 . La fórmula es la siguiente:

Robustez $=((\mathrm{N}-\mathrm{E} 1-0.3 \mathrm{E} 2) / \mathrm{N}) * 100$

c. Innovación.- Es un conjunto de atributos que a pesar no fueron determinados por los usuarios,

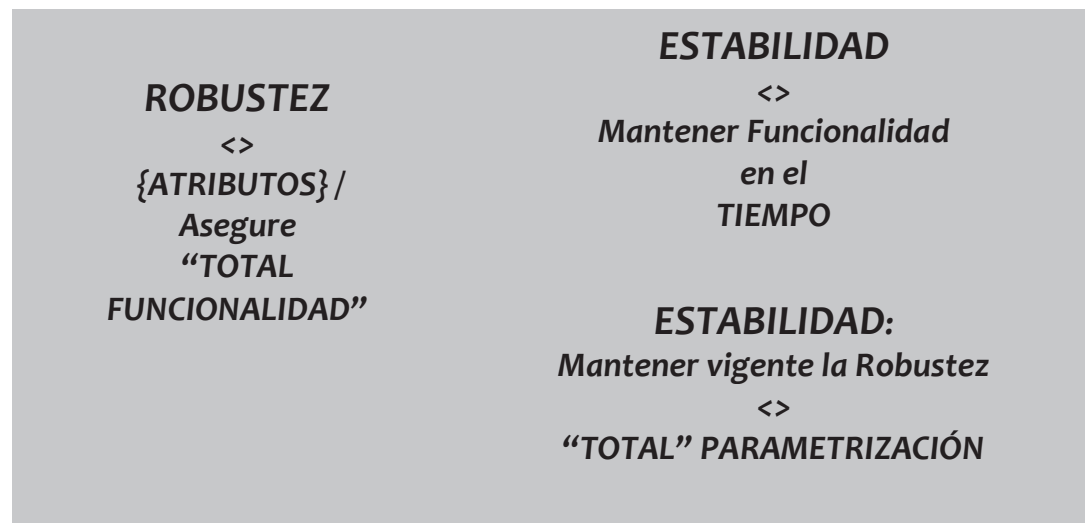

Figura 3. Características básicas de todo modelo [4].

el modelador los define en función de su concepto sobre el negocio y su pertinencia. Una estrategia conocida es la generalidad, vale decir que el esquema sea válido para toda la casuística posible.

d. Estabilidad.- La facilidad con la que el modelo de datos puede reflejar cambios en los requisitos sin cambiar el modelo de datos en sí. La flexibilidad es una de las características más importantes de un modelo de datos (Avison y Fitzgerald 1995, Leviten y Redman 1995) y tiene un efecto importante en los costos de mantenimiento de un sistema (Moody y Shanks 1994). El uso de conceptos genéricos y la representación de las reglas de negocio como las estructuras en el modelo de datos darán lugar a un modelo de datos más flexible (Barker 1989, Simsion 1994).

\section{Comentario}

Se han presentado las características más relevantes de todo modelo conceptual. Cualitativamente las tres últimas son claves, porque ellas aseguran Robustez y Estabilidad. Figura 3.

Como trabajo de investigación se atacará frontalmente estos temas con el objetivo de llegar a indicar el Cómo hacerlo. El aporte de este artículo radica en indicar las características fundamentes presentes en toda Arquitectura de Datos: Robustez y Estabilidad.

Los Modelos conceptuales son la base de todo modelo de datos. En el estado de gestación del sistema se plasman las características fundamentales, entre 
ellas surge la información contextual, es decir aquella que es relevante para el sistema y, sobre todo para el consumidor de información.

De allí que el contextualizar es una exigencia hoy en día. Para lograr esta materialización se puede optar por varias opciones factibles, como crear una capa de software que contenga el perfil de cada una de las instancias de la clase Cliente entre otras.

La propuesta desarrollada por el autor, propone ampliar las dependencias entre atributos que posee el modelo Relacional. La nueva Dependencia es la Contextual.

Luego como consecuencia en el proceso de normalización de la base de datos, al aplicar el análisis de Dependencia este tendrá la siguiente secuencia:

Análisis de dependencia.- Funcional, Transitiva, Múlti-Valuada y la Contextual. Por ejemplo si se desea conocer las condiciones atmosféricas, el sistema debe ser capaz de determinar el punto geográfico que el consumidor de la información (cliente) le pide y la fecha-hora de la que se desea conocer. En este ejemplo es clara la dependencia del dato con el tiempo en este caso la fecha-hora.
Ejemplo de atributos con dependencia contextual: qué tipo de ropa debería vestir para determinada ocasión, los valores son múltiples dependiendo del tipo de ocasión con tipo de ropa.

\section{Comentario}

Para materializar la Dependencia Contextual se debe plantear la Relación entre la variable independiente (contexto) y la variable o atributo dependiente (varia con como varía el contexto).

La variable de contexto puede tener diferente naturaleza, sin embargo funciona como el marco de referencia sobre el consumidor de la información.

Por ejemplo en un típico evento de una Venta, los productos a ser presentados a los clientes deberán ser de acuerdo a su perfil histórico de compras, luego dado un cliente define un perfil del cliente y, en base a su perfil se determina la oferta a realizar. Se ha descubierto una relación entre oferta y perfil. La oferta varía con el perfil dado. Ver Figura 4.

En general todo consumidor de información genera su propio perfil en el transcurrir del tiempo a través de eventos cotidianos a nivel del negocio.

\section{comportamientos}

\begin{tabular}{c|l}
\hline & Entidad-Alumno: \\
\hline Entidad-Cliente: & Atributos: \\
\hline Atributos: & .Código-Alumno \\
\hline Nro_Cliente & .Fecha_Ingreso \\
\hline .Fecha_Ingreso & .Total_Cursos_matriculados \\
.Total_Compras & .Total_Cursos_aprobados \\
.Total_Monto & .Total_Créditos_aprobados \\
.Total_Compras_Créditos & .Total_Créditos_Obligatorios \\
.Total_Compras_Contado & .Record_Asistencia \\
.Total_Compras_Internet & .Promedio_Ponderado \\
.Total Compras_Directas & .Estado \\
\hline .Estado & \\
\hline
\end{tabular}

Los atributos son generados por los eventos consumados. 
Proceso = Análisis de Datos

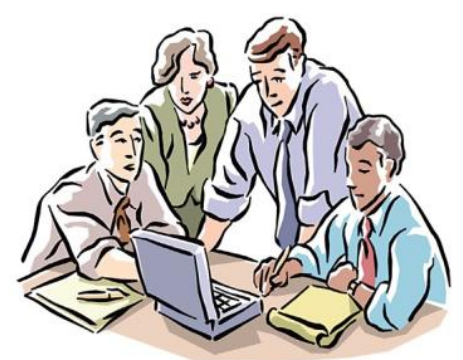

Proceso de Calidad

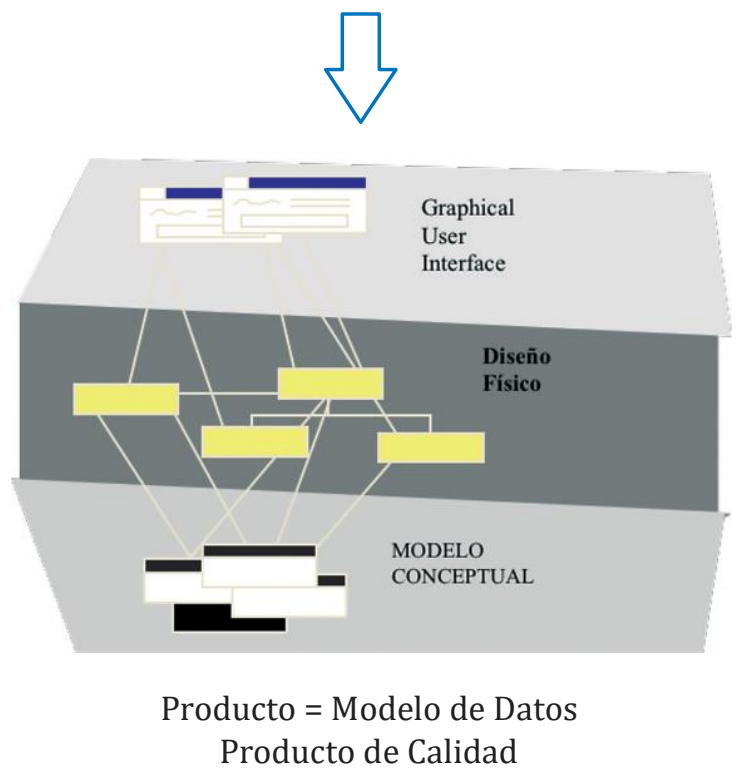

Figura 5. Proceso de análisis de Datos.

Los perfiles son consecuencia del comportamiento manifiesto en el transcurrir del tiempo. Por cada evento consumado se debe generar un acumulado del contexto es decir si el evento se trata de una venta, los acumuladores deberían ser lo comprado por el cliente sea por Internet, teléfono o presencial, al contado o crédito, etc.

De la configuración de totales o acumuladores se obtiene el perfil y en su siguiente compra el sistema deberá contextualizar el evento reproduciendo las condiciones más típicas asociadas al cliente.

\section{Métodos de Modelado de Datos}

El principal problema a investigar en este artículo es evaluar la calidad de un modelo de datos de un sistema de información de gran volumen de datos. Como problemas secundarios se tiene el rediseño de procesos de datos, determinar la diferencia de calidad de modelado entre noveles y expertos. Finalmente la automatización del proceso de evaluación.

La idea tradicional de la calidad del software ha sido la de recurrir a la "fuerza bruta" pruebas en desarrollo. Sin embargo, el enfoque de Gestión de la Calidad Total (TQM) sugiere que es más rápido y eficiente concentrar los esfuerzos durante las primeras fases de desarrollo de un producto, con el fin de detectar y corregir defectos tan pronto como sea posible.

Los estudios empíricos han demostrado que el traslado de esfuerzo de calidad hasta las fases tempranas del 
desarrollo puede ser 33 veces más rentable. La etapa de análisis se toma como un arte más que una disciplina de ingeniería, y la calidad es por lo tanto más difícil de evaluar. Hay relativamente pocos esquemas para la evaluación de la calidad de los modelos de datos, y poco acuerdo incluso entre los expertos en cuanto alo que hace un "buen"' modelo de datos. Como resultado, la calidad de los modelos de datos producidos en la práctica es casi totalmente dependiente de la competencia del modelador de datos.
La calidad del proceso se centra en el proceso usado para producir el producto. En nuestro caso el producto es el modelo de datos. El objetivo es construir calidad en el proceso de producción en lugar de tratar de añadir al final a través de revisiones e inspecciones del producto acabado. El enfoque de la calidad del proceso está en la prevención de defectos en lugar de detección, para reducir y minimizar la dependencia de inspecciones de masas como una forma del logro de la calidad.

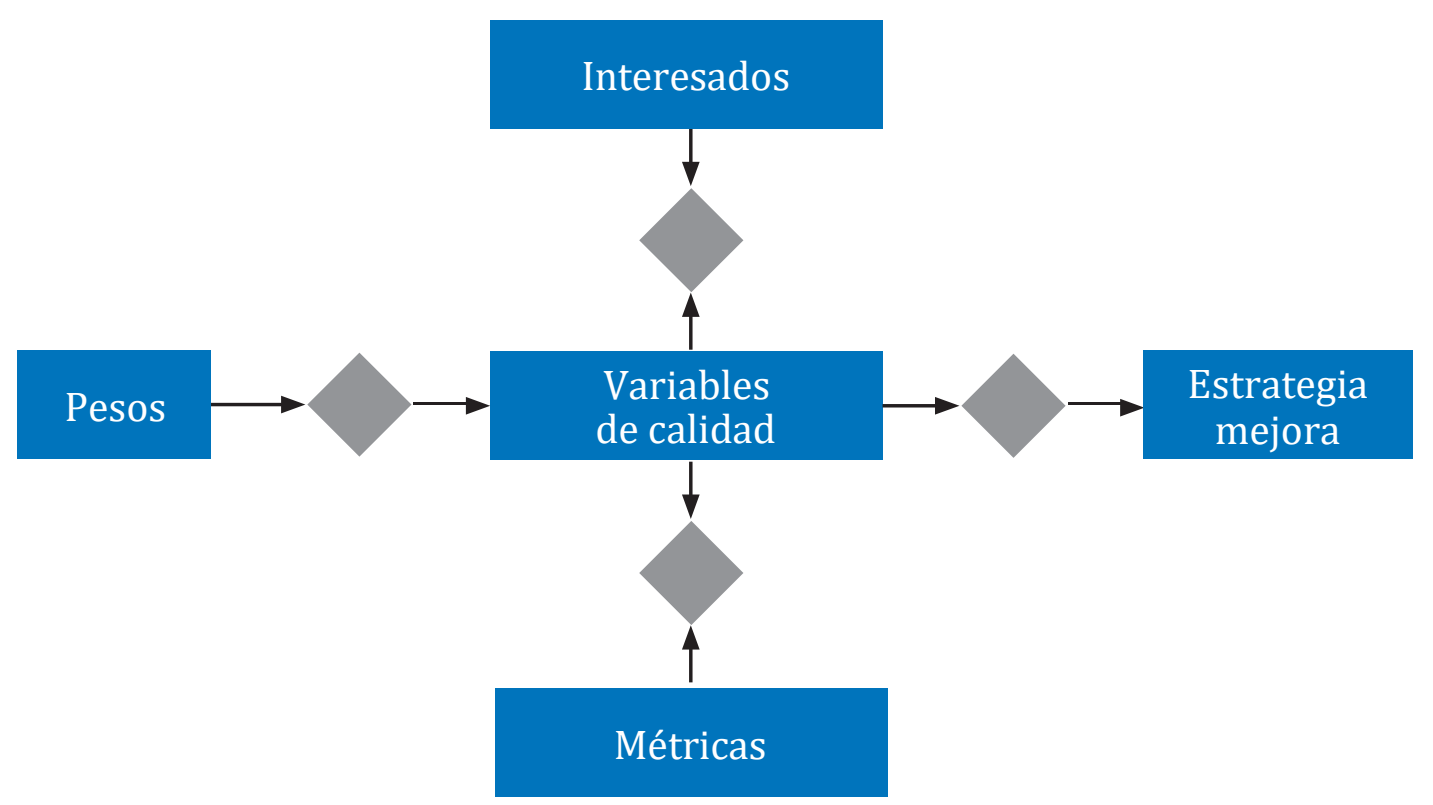

Figura 6. Componentes del método.

Desde el punto de vista teórico basado en la semiótica el modelo conceptual debe cumplir por lo menos con tres dimensiones de calidad: sintáctico, semántico y pragmático.

El modelo propuesto de validación de calidad está compuesto por cinco variables: Figura 6.
1. Personas Interesadas: Área de negocios y área técnica

2. Características de Calidad:

Completo (robustez) cuando el modelo posee todos datos que pueda requerir el negocio Flexibilidad: Utilizando parámetros.

3. Ponderación de las características: Definir pesos 
para cada uno de los factores mencionados.

4. Métricas: Criterios de medición

5. Estrategias de mejora

\section{ESQUEMA DE INVESTIGACIÓN: INVESTIGACIÓN EN ACCIÓN}

Se aplicó este método para sumar los conocimientos académicos y prácticos y así salvar los inconvenientes de teorías interesantes pero no aplicables en la práctica y conocimiento práctico validado con la experiencia real. Este método se desarrolla en forma espiral de manera sistemática: se planifica se valida, con el resultado obtenido se repite el cicloplanificar-validar.

Debido a que está orientada a la acción, es especialmente adecuado para la validación de conocimientos cómo métodos. Es muy conveniente para una investigación en Ingeniería. Los resultados reales no fueron exitosos en principio se detectó falta de robustez, atributos innecesarios, data redundante de sistemas vecinos (falta de demarcación).

\section{Comentario}

Los aportes principales son dos:

Presentar las características para determinar la calidad de un modelo de datos y la segunda es el método utilizado que para ingenieros es una actividad natural durante nuestro diario desarrollo profesional.

En lo que respecta las fuentes de datos para modelar es una investigación que depende en gran medida del aporte de los interesados como los usuarios. Lo cual es una dependencia que podría asegurar sólo la satisfacción del usuario puntualmente en un ámbito de tiempo y punto de vista, porque para nuevos usuarios no necesariamente se mantendrían los mismos requerimientos. Inclusive para los mismos usuarios en tiempos futuros podrían sentirse insatisfechos por la falta de información no considerada en su etapa inicial [6].

Este documento trata de proporcionar el modelo de conocimiento necesario para facilitar la captura de información de la transacción y estructurarla, para almacenarla pero no solo la data final, sino por el contrario el detalle de los eventos que se dan en el día a día en los negocios. De esta se obtendría en detalle la información completa en todo el flujo de la transacción.

Todo evento es rico en información por que describe toda la circunstancia, variables, factores y consecuencias que giran al entorno de los eventos. La información proporcionada por las transacciones nos da la inteligencia de negocios de manera estructurada. Porque en base a los eventos conocemos el comportamiento de los entes de manera dinámica. Por cada evento cambia el comportamiento de los entes involucrados en la transacción. Si bien es cierto este cambio puede ser un pequeño diferencial sin embargo en el tiempo su acumulado indicará marcadamente comportamientos particulares de los objetos pertenecientes a la misma clase.

El artículo se basa en el modelo REA que fue propuesto por McCarthy en 1982, el cual plantea modelar basándose en tres tipos de entes básicos Recursos, Eventos y Agentes. En su época McCarthy lo aplico exclusivamente al dominio contable. Basado en este esquema el autor lo amplía con otros componentes.

En base al trabajo de McCarthy el autor le adiciona más entes fundamentales como, las actividades futuras todo ente que participe en la transacción o evento. Los elementos adicionales son los Facilitadores, Uso y Resultados de manera muy amplia y genérica de manera que se pueda adaptar a cualquier sistema en particular. De esta manera se plantea un modelo REA extendido, Figura 6.

El ciclo de vida de una Transacción se define en términos de una Ontología REA extendida. La cual da conocimiento sobre la transacción que está siendo investigada. Los eventos están comprometidos de manera que establecen un impacto en cambios económicos y transaccionales (de manera dual).

\section{Comentario}

El aporte de este artículo está en la intención de generalización del modelo básico sin embargo la implementación debe ser replanteada con ajustes conceptuales basados tanto en la semántica como en la ontología. Por ejemplo entidades como Resultado no debería usarse dado que el resultado es o un cambio u creación de alguna instancia de la entidad en el modelo. Por lo que el aporte es más conceptual que concreto, sin embargo abre un horizonte para completar el modelo original REA. Figura 7.

Lo que no se detalla es la manera de llegar a constituir el dominio de datos o atributos necesarios para iniciar la síntesis del modelado, es un vacío que deberá ser cubierto en la investigación en curso.

Hasta aquí se cuenta con una parte fundamental de la Arquitectura que lo constituyen sus componentes como:

- Personajes 


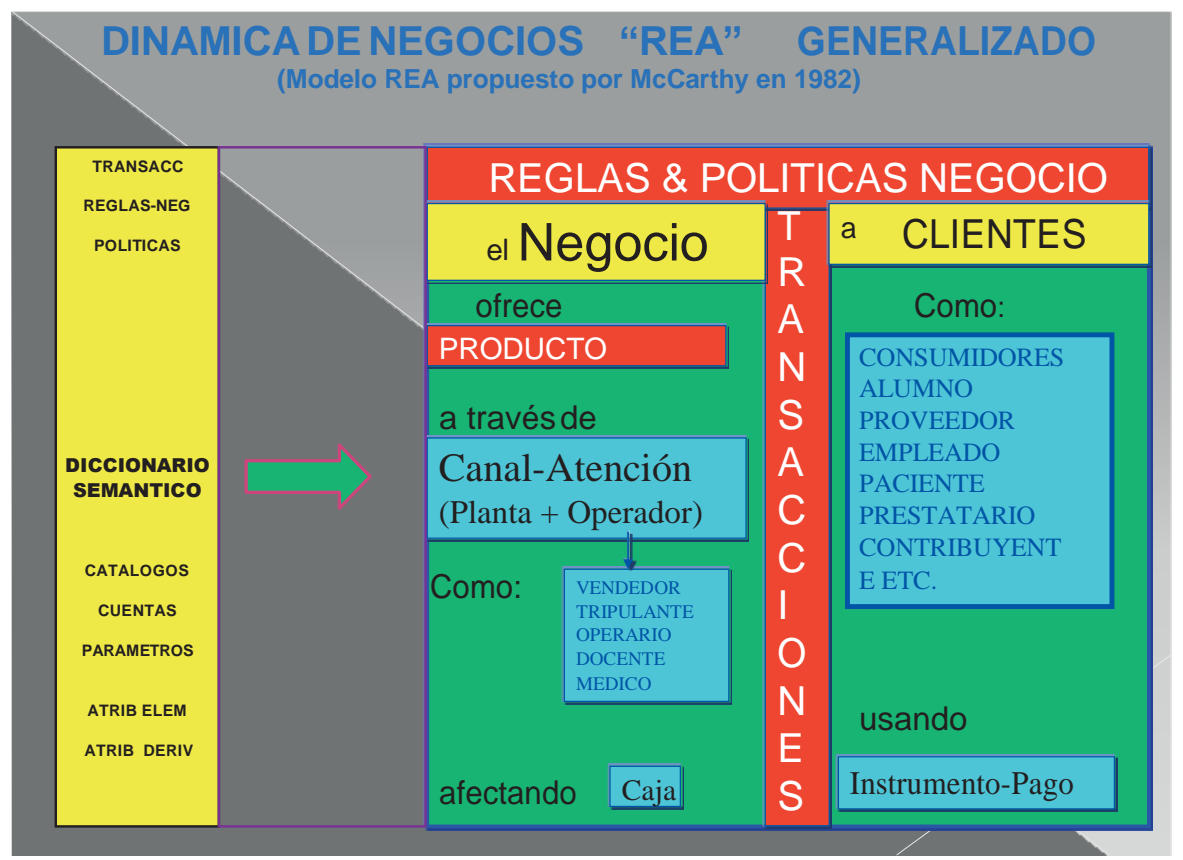

Figura 7. Modelo REA generalizado[7]

- Productos

- Transacciones

- Políticas de Negocio

- Reglas de Negocio

- Canales de Atención

En el proceso de diseño de un artefacto de software, uno de los primeros pasos es construir un modelo de datos que representa necesidades de los usuarios. Este modelo conceptual de datos se convierte en parte de la base del sistema informático.

Colocación de las entidades de datos en tres tipos de entidades que representa los recursos económicos, los eventos y los agentes y estableciendo la secuencia de eventos la que típicamente se producen en un contexto de negocios. Esto tiene importantes implicaciones prácticas y teóricas:

En primer lugar, si profesionales nóveles cuenta con una mejora significativa en la realización básica de tareas de base de datos, generarán beneficios para la organización mediante la mejora de su rendimiento.

En segundo lugar, se extiende resultados anteriores mediante el examen del tema en que los métodos de modelo de datos impactan subsecuentes en la comprensión y evaluación del sistema.

No existen esquemas de trabajo estándar o general a seguir para la colocación de las entidades en el modelo ER.

Algunos investigadores han propuesto la estructuración de los diagramas ER por la incorporación de dominio semántico específico (por ejemplo, contabilidad recursos, eventos, agentes, (REA)) para mejorar la representación semántica. Un aspecto interesante de esta semántica es la sustentación de su esquema.

El modelo REA propuesto por McCarthy en 1982, es correcto pero limitado a un dominio contable. Luego lo que sigue es poder generalizarlo y completarlo con algunos elementos adicionales que representen de manera general a toda empresa.

Se llega a establecer una organización del modelo que refleja la realidad de manera clara y coherente. Con el sentido semántico será posible visionar los procesos implícitos insinuados por los datos y sus relaciones entre ellos.

En este experimento se comprueba si un modelo está sintácticamente correcto o no, mas no es posible validar el nivel de su contenido para lograr su Robustez semántica que satisfaga toda la funcionalidad del negocio. Se puede considerar un experimento orientado a nivel de diseño.

\section{Comentario}


La investigación comprobó que el esquema REA generalizado, es sólido y permite operarlo sin mayor complicación por profesionales nóveles con poca experiencia constituyéndose en una excelente guía de análisis o lista de validación de componentes. Este es el esquema que deberá consolidarse como el universal a nivel demodeladodeArquitecturaparaun proyectoInformático.

\section{CONCLUSIONES}

Se han tratado seis artículos de muchos otros, los cuales aportan conceptos importantes para el modelado de la Arquitectura de datos. Como entender la Naturaleza de Modelar, determinar la variable de Robustez como la característica más preponderante en esta etapa, la necesidad de considerar la Dependencia Contextual de la información (principio de la relatividad del dato), reconocer los componentes que deben estar presentes en toda Arquitectura, la importancia de la etapa de modelado en la calidad final del software.

\section{REFERENCIAS}

[1] Boehm B W 1981 Software Engineering Economics Prentice-Hall:New Jersey

[2] Graeme S, Simon K M and Graeme S 2012 Data modeling: Description or design? Information \& Management

[3] Conceptual Data Modelling 1997 An Empirical Study of Expert and Novice Data Modellers Australia

[4] Choi D, Kim N, Hung D T 2012 Conceptual data modeling for realizing context-aware services Expert Systems with Applications 39(3) pp 3022-3030

[5] Moodya D L and Graeme G S 2002. Improving the quality of data models: empirical validation of a quality management framework

[6] Biscontri R G 2007 Linking Knowledge to Accounting Transactions: A Database Approach

[7] Fuller R M, Uday M B and Schafer B A The effects of data model representation method task performance 